\title{
Clinical Management of Traumatic Hyphema
}

\author{
G.S.Haritha, G.Saritha and K.Nalini Kumari
}

\begin{abstract}
Hemorrhage in the anterior chamber of the eye, or hyphema, results from a breakdown of the bloodocular barrier $(B O B)$ and is frequently associated with inflammation of the iris, ciliary body, or retina. Hyphema attributable to blunt or perforating ocular trauma is more common than that resulting from endogenous causes. The clinical appearance of hyphema is variable and is influenced by the volume of blood and the amount of time erythrocytes are present in the anterior chamber. When hyphema is evident, a complete history should be obtained and a thorough physical examination performed to direct the initial selection of diagnostic tests. The two primary management issues in animals with hyphema are prevention of secondary hemorrhage (by treating the underlying disease) and control of secondary glaucoma.
\end{abstract}

Keywords: Traumatic, Hyphema, clinical management

\section{Introduction}

Hyphema, or hemophthalmos is defined as hemorrhage within the anterior chamber of the eye. There are numerous potential intraocular causes for hyphema, such as blunt or penetrating trauma, inflammation, retinal detachment, primary or metastatic neoplasia, iatrogenic procedures, congenital anomalies, and chronic glaucoma (Tessie and Claudio, 2015). When trauma has been eliminated as a possible cause, it is prudent to assume that every animal with hyphema has a serious systemic disease until proven otherwise (Andras et al., 1999). Hyphema may also result from systemic diseases, including widespread inflammation and vasculitis associated with infectious diseases, severe acute anemia, systemic hypertension, and disorders of hemostasis like coagulopathies and hyperviscosity syndromes (Tessie and Claudio, 2015). When hyphema is evident, a complete history should be obtained and a thorough physical examination performed to direct the initial selection of appropriate diagnostic tests. The prognosis for animals with hyphema depends on identifying the underlying cause; initiating proper treatment; and careful, long-term follow-up (Andras et al., 2000). The present paper discusses about the management of hyphema due to trauma.

\section{Materials and Methods}

A one year-old, male mixed breed was presented with history of redness of left eye. Owner reported that the dog had a fight with a stray dog that lives near their locality. The following day, the owner noticed redness of anterior chamber of eye. During a thorough ophthalmic examination, a pool of blood in the left eye anterior chamber approximately on the anterior pupillary iris was noticed. A thorough physical examination was also carried out to detect any other injuries/ underlying causes. However, trauma was the primary cause of hyphema in present case. Detailed ophthalmic examination of left eye revealed presence of sluggish menance and dazzle reflex, pupillary light reflex is not evident as the hyphema was covering the anterior pupillary iris. Whereas, reflexes were clearly present in the right eye. Resorption of blood, which requires therapeutic management, may help in further ophthalmic examination to rule out retinal status. The intraocular pressure measured by schiotz tonometer was found to be normal for both eyes i.e., $19 \mathrm{~mm} \mathrm{Hg}$ (left eye) and $17 \mathrm{~mm} \mathrm{Hg}$ (right eye). Based on anamnesis, ophthalmic examination it was diagnosed as traumatic hyphema (mild degree).

\section{Treatment and Discussion}

Mild degree of hyphema can actually be resorbed over a period of days to weeks depending on the amount of blood present. Topical ocular application, Eye drops antibiotic with steroid i.e., Onemox-DM® (Morbifloxacin and 1\% Dexamethasone), and cycloplegic drug i.e., Oculate ${ }^{\circledR}$ (Cyclopentolate Hcl 1\%) was advised. Corticosteroids are prescribed to reduce the inflammation and cycloplegic drugs are used to improve patient comfort and prevent the formation of posterior synechia. Steroids stabilize the blood-ocular barrier and directly inhibit fibrinolysis. Cycloplegics are anticholinergic drugs. They temporarily inhibit acetylcholine receptors in the iris sphincter muscle and ciliary body. Cycloplegic drug helps in dilatation of pupil and enhances resorption of blood (Nalinee, 2007). In addition, both cycloplegics and corticosteroids may reduce the risk of secondary hemorrhage. Cycloplegics minimize iris movement and stress on the original ruptured vessels. Management is very important in order to prevent self trauma/ rubbing of eyes. Hence, application of elizebethan collar was advised. Elevation of head posture is advised as it promotes more rapid blood resorption and lowers venous pressure to the globe, helping to reduce IOP and to allow for clot formation and resolution (Priscilla and Dorothy, 2014). It is very important that the dog's activity is restricted to minimize the possibility 
of stimulating the recurrent or secondary hemorrhage. After nine days of therapy improvement was noticed, as retracted blood clot towards the lower aspect of globe. The menance, dazzle and pupillary light reflexes were present in left (affected) eye at day 9. The presence of a PLR is a favorable prognostic indicator for retinal function and the potential for vision (Ron, 2015). The common complications following hyphema are posterior synechia, peripheral anterior synechia, corneal blood staining and optic nerve atrophy with chronically elevated intraocular pressure (Nalinee, 2007). The owner was warned about the complications and was advised to report if any complication is noticed. However, complete resolution of blood clot was not observed.

\section{Conclusion}

The most common mode of clinical presentation in blunt ocular trauma is hyphema. When hyphema is noticed, a detailed history and thorough clinical examinations are necessary to rule out the cause, select appropriate treatment and predict visual outcome. Hyphema does not typically cause permanent loss of vision; however, its presence signifies considerable insult to the globe and therefore requires careful follow-up and management. Patient education is essential to minimize complications in the first several days after the injury as well as for the long-term ocular health of the patient. Recurrence of bleeding should be monitored shortly after the hyphema is identified regardless of its severity. A monitoring of an elevation of the intraocular pressure is strongly recommended.

\section{Reference}

[1]. Andras M. Komaromy, David T. Ramsey, Dennis E. Brooks, Cynthia C. Ramsey, Maria E. Kallberg and Stacy E. Andrew, 1999. Hyphema. Part I. Pathophysiologic Considerations. Compendium on Continuing Education for the Practicing Veterinarian, 21 (11), 1064-1069.

[2]. Komaromy, A. M., Ramsey, D. T., Brooks, D. E., Ramsey, C. C., Kallberg, M. E. and Andrew, S. E. 2000. Hyphema. Part II. Diagnosis and Treatment. Compendium on Continuing Education for the Practicing Veterinarian, 22 (1), 74-79.

[3]. $\quad$ Nalinee Tuntivanich. 2007. Ophthalmology Snapshot. Thai J. Vet Med. Vol. 37(4): 51-52.

[4]. Priscilla Lenihan and Dorothy Hitchmoth. 2014. Traumatic Hyphema: A Teaching Case Report. Optometric education. Volume 39, Number 3 / Summer 2014.

[5]. Tessie Beck Martins and Claudio S.L. Barros, 2015. Red eyes in the necropsy floor: twenty

[6]. cases of hyphema in dogs and cats. Pesquisa Veterinária Brasileira 35(1):55-61.
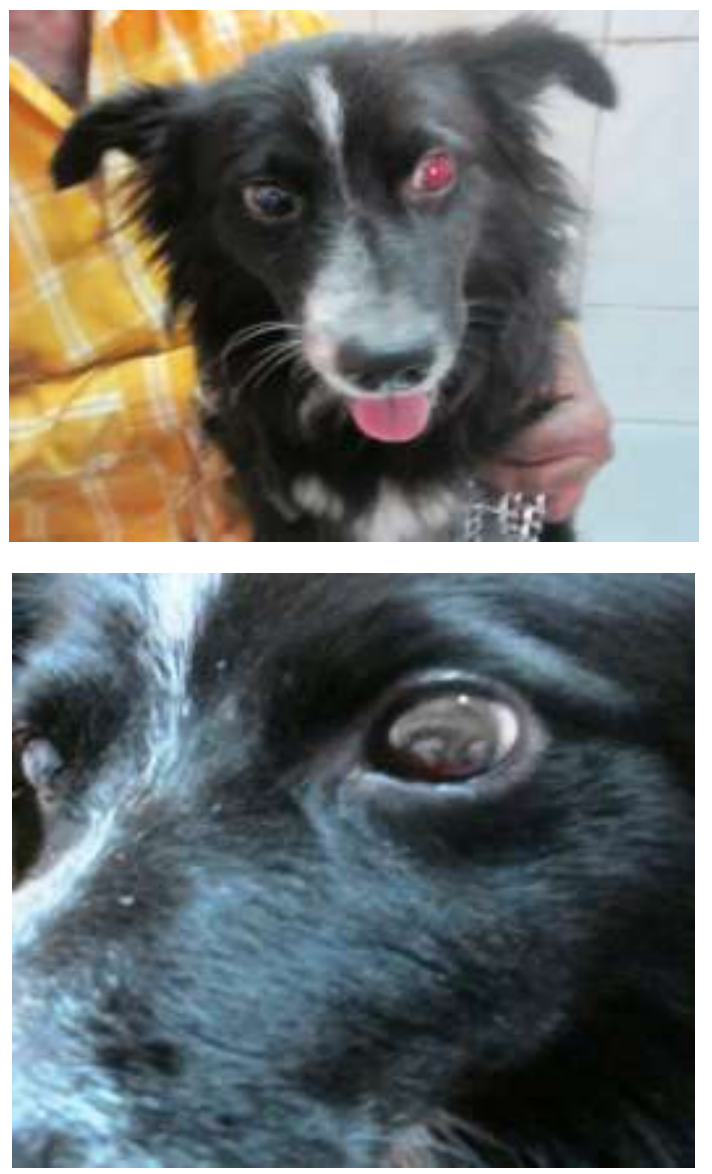